INVESTIGACIÓN

Recibido: 28/05/2020 --- Aceptado: 15/12/2020 --- Publicado: 12/03/2021

\title{
LA ATENCIÓN A LA DIVERSIDAD DURANTE LA COVID-19: REVISIÓN LEGISLATIVA DE LAS MEDIDAS SEGÚN LOMCE
}

\author{
Attention to diversity during COVID-19: Legislative review of measures \\ according to LOMCE
}

D 87 $\mathbb{R}^{\mathrm{C}}$ Javier Rodríguez Torres': Universidad de Castilla-La Mancha. España Javier.rtorres@uclm.es

(8) $\mathbb{R}^{6}$ Óscar Gómez Jiménez: Universidad de Castilla-La Mancha. España. osquitagomez@gmail.com

\section{RESUMEN}

Tras el establecimiento del estado de alarma provocado por la COVID-19 y, el consiguiente cierre de los centros educativos a nivel nacional, la educación formal se ha trasladado de un contexto de enseñanza presencial a entorno virtuales de aprendizaje en cuestión de horas. En este trabajo, presentamos una revisión de la normativa educativa española sobre la atención a la diversidad del alumnado y de las medidas a aplicar en contextos extraordinarios de aprendizaje, como el que vivimos a nivel mundial. Para su realización, hemos realizado un análisis de los contenidos, centrado en las medidas de atención a la diversidad existentes en la legislación orgánica nacional y en las directrices de aplicación del Ministerio de Educación y Formación Profesional. Las políticas educativas, y las administraciones responsables, no se encuentran preparadas para un cambio en la metodología de atención al alumnado, que incida en la disminución de las desigualdades del alumnado y proporcione un acceso equitativo al aprendizaje, ya que el rol compensador de la escuela (como institución) no puede suplirse a través de la virtualización de la enseñanza. En este aspecto, los docentes y la autonomía de los centros suponen un valor añadido en la igualdad de oportunidades, el cual se ve disminuido en entornos virtuales educativos por las condiciones socioeconómicas del alumnado, aumentando la desigualdad educativa

\footnotetext{
${ }^{1}$ Javier Rodríguez Torres: Doctor en Pedagogía. Profesor en la Facultad de Educación de Toledo. En la actualidad coordinador de campus del Máster de Secundaria. Las líneas prioritarias de investigación: atención a la diversidad, TIC, elaboración de materiales, género y currículum.
} 


\title{
LOMCE
}

PALABRAS CLAVE: Atención a la diversidad - Educación inclusiva - Atención educativa - Legislación educativa - COVID-19 - Necesidades educativas - Inclusión - Escuela Inclusiva.

\begin{abstract}
Following the establishment of the state of alarm caused by COVID-19 and the consequent closure of schools nationwide, formal education has moved from a presential learning context to virtual learning environments in a matter of hours. We present a review of the Spanish educational regulations on attention to students' diversity and the measures to be applied in extraordinary learning contexts, such as the one we live worldwide. For the realization, we have done and analysis of the contents, focus on the measures of attention to the diversity existent on the national organic legislation and on the guidelines of the Education and Vocational Training Ministry. The educational policies, and the responsible administrations, are not prepared for a change in the methodology of attention to the students, that affects in the reduction of the inequalities of the students and provides and equitable access to learning, since the compensatory role of the school (as an institution) cannot be supplied through the virtualization of teaching. In this aspect, teachers and the autonomy of the centers represent and added value in equal opportunities, which is diminished in virtual educational environments due to the socioeconomic conditions of the students, increasing educational inequality.
\end{abstract}

KEY WORDS: Attention to diversity - Inclusive education - Educational attention Educative legislation - COVID-19 - Educational necessities - Inclusion - Inclusive School.

\section{A ATENÇÃO A DIVERSIDADE DURANTE A COVID-19: REVISÃO LEGISLATIVA DAS MEDIDAS DE ACORDO A LOMCE}

\section{RESUMO}

Depois do estabelecimento do estado de alarme provocado pela COVID-19 e, o consequente fechamento dos centros educativos a nível nacional, a educação formal foi transformada de um contexto de ensino presencial para entornos virtuais de aprendizado em questão de horas. Neste trabalho, apresentamos uma revisão da normativa educativa espanhola sobre a atenção à diversidade dos estudantes e das medidas a serem aplicadas em contextos extraordinários de aprendizagem, como este que vivemos a nível mundial. Para sua realização, fizemos uma análise dos conteúdos, focando nas medidas de atenção à diversidade existentes na legislação orgânica nacional e nas diretrizes de aplicação do Ministério de Educação e Formação Profissional. As políticas educativas, e os entes responsáveis, não estão preparadas para uma mudança na metodologia de atenção aos alunos, que implique a diminuição das desigualdades dos alunos e proporcione um acceso equitativo ao aprendizado, já que o papel compensador da escola (como instituição) não pode ser 


\section{LOMCE}

fornecido através da virtualização do ensino. Nesse aspecto, os professores e a autonomia dos centros têm um valor adicionado na igualdade das oportunidades, $\mathrm{o}$ que se vê diminuído em entornos virtuais educativos pelas condições socioeconómicas dos alunos, aumentando a desigualdade educativa.

\section{PALAVRAS CHAVE}

Atenção a diversidade - Educação inclusiva - Atenção educativa - Legislação educativa - COVID-19 - Necessidades educativas - Inclusão - Escola Inclusiva

\section{Cómo citar el artículo:}

Rodríguez Torres, J. y Gómez Jiménez, O. (2021). La atención a la diversidad durante la COVID-19: revisión legislativa de las medidas según LOMCE.Vivat Academia. Revista de Comunicación, 154, 1-19. http://doi.org/10.15178/va.2021.154.e1241

http://www.vivatacademia.net/index.php/vivat/article/view/1241

\section{INTRODUCCIÓN}

La situación educativa española se encuentra en un momento excepcional, debida a la crisis internacional producida por la COVID-19. El 13 de marzo de 2020 se anunciaba el cierre de los centros educativos y el comienzo del confinamiento de la población española en sus hogares (BOE, 2020a; EFE, 2020).

Los centros educativos se encuentran con una situación sobrevenida, para la que ningún centro se encuentra preparado: la virtualización de la enseñanza por la clausura de los centros educativo por una duración (aunque principalmente se estableció por quince días) indeterminada.

En ese momento, las administraciones educativas (tanto autonómica como centrales) comienzan una carrera a contra reloj para establecer las normas de funcionamiento de los centros durante el confinamiento de la población española, así como el seguimiento de las clases a través de medios y dispositivos de los centros educativos (BOE, 2020b). Los docentes se ven obligados a cambiar sus aulas por sus espacios domésticos, convertidos en improvisadas aulas, donde seguir impartiendo los contenidos de un segundo trimestre prácticamente finalizado (cuyas evaluaciones se encontraban completadas) y con la esperanza de retomar la presencialidad después del periodo festivo de Semana Santa.

Un despliegue de plataformas educativas (Juste, 2020) pusieron sus recursos disponibles para la comunidad educativa (de manera gratuita en la mayoría de los casos) y los docentes tuvieron que desplegar sus competencias digitales para una transformación educativa de la que no se estaba preparado (a pesar del intento de transformación digital de la gran mayoría de los centros, que se encontraba comenzada, en fases iniciales o medias, o por comenzar). 


\section{LOMCE}

Dado que nos encontramos ante una sólida comunidad educativa, docentes y familias aprendieron a digitalizar su concepto sobre la educación y se empezaron a establecer canales y plataformas educativas donde los alumnos pudieran acceder al seguimiento de sus clases, entrega de trabajos y comunicación directa con los profesores. Todo ello, sumado a la disposición de recursos tecnológicos personales del profesorado (Cueto, 2020) y de las familias, lo cual puede llegar a suponer el establecimiento de una nueva categorización del alumnado en función de sus recursos de acceso y de seguimiento educativo, durante el confinamiento y desescalada de la crisis social y educativa provocada por el SARS-CoV-2.

Esta nueva situación educativa, destacada por opiniones, sociales y profesionales, diversas sobre el modelo educativo y la digitalización de la enseñanza de una manera apresurada y sin la debida formación por parte de la comunidad educativa (Martín \& Rogero, 2020), centra los esfuerzos en la continuidad educativa del gran porcentaje del alumnado y en la transformación de las planificaciones educativas del modelo presencial, dejando en un impasse la atención a la diversidad del alumnado, debido a la urgencia de la transformación de la actividad lectiva presencial a los entornos educativos virtuales.

\subsection{La atención a la diversidad del alumnado.}

La situación actual sobrevenida nos hace replantear el momento en el que se encuentra la atención a la diversidad del alumnado y que medidas se han de tomar, partiendo de lo establecido en los preceptos nacionales. Antes de comenzar a analizar las medidas de atención a la diversidad que plantea la legislación educativa española, es necesario definir el concepto de estudio. Entendiendo la atención a la diversidad como el conjunto de acciones educativas que dan respuesta a las necesidades del alumnado del centro y que requieren de una actuación específica de factores personales o sociales (Rodríguez y Gómez, 2018), es necesario la adaptación del entorno educativo a las necesidades educativas especiales a través de nuevas prácticas profesionales que conlleven la creación de actividades centradas en el pleno desarrollo de la persona y de sus necesidades personales y sociales.

Para ello, y haciendo alusión al concepto de escuela inclusiva, se han de crear programas educativos específicos que tengan en cuenta la diversidad, las características y las necesidades del alumnado, permitiendo su integración dentro de la comunidad educativa y proporcionando un rol participativo y preponderante en su proceso de aprendizaje e inclusión, así como potenciar su participación social dentro del grupo de iguales en los que se encuentra el alumnado (Espinosa, Reyes y Rodríguez, 2015).

Dicha inclusión educativa se ha de alcanzar a través de la creación de herramientas y espacios de participación común a todos los alumnos (independientemente de sus características personales) y debe promover la disminución de todos los procesos que conlleven la exclusión del alumnado (Barton, 


\section{LOMCE}

2009), participando y tomando parte activa en la vida del centro, ya que son el núcleo central de la educación.

La educación tiene como objetivo la promoción del desarrollo y de la socialización de todo el conjunto del alumnado, sin excepciones y bajo la amplitud y profundidad como sea posible, ya que la atención a la diversidad no es una opción, sino una parte insoslayable que requiere ser cumplido en todos sus aspectos, dando sentido a la acción educativa en sí misma. El alumnado, en su conjunto, es un gran potencial en el aprendizaje, ya que la heterogeneidad de este supone un valor añadido en la atención a la diversidad por sus distintas capacidades, culturas y necesidades.

La escuela, y su función compensadora, rompe con las desigualdades familiares y sociales del alumnado, ya que considera a su totalidad del alumnado en la construcción de lo común y permite el desarrollo equitativo de las competencias, habilidades y destrezas de los alumnos, sobre todo desde el punto de vista del desarrollo del bienestar emocional, físico y personal del alumnado (Díez y Gajardo, 2020).

Estas concepciones previas que hemos expuesto quedan recogidas en el preámbulo de la Ley Orgánica 2/2006, de 3 de mayo, de Educación, modificada por el articulado único de la Ley Orgánica 8/2013, de 9 de diciembre, para la Mejora de la Calidad Educativa (en adelante, LOMCE). Este preámbulo establece que la educación supone facilitar el desarrollo personal y la integración social, determinando las metas y expectativas de la trayectoria vital, así como el conjunto de conocimientos, recursos y herramientas de aprendizaje que capacitan a una persona para cumplir con éxito sus objetivos. Esto se ha de garantizar a través de los principios del sistema educativo, dado que la educación debe garantizar la calidad de la educación para todo el alumnado, la equidad, la igualdad de derechos y de oportunidades, la no discriminación, la inclusión educativa, la flexibilización y la personalización de la educación, así como la orientación educativa y profesional, entre otros (BOE, 2010).

La atención a la diversidad en educación ha de regirse bajo los principios de normalización e inclusión, garantizando la no discriminación y la igualdad efectiva en el acceso y permanencia en el sistema educativo, así como ha de prestar especial atención a las desigualdades derivadas de cualquier tipo de discapacidad. Las administraciones educativas han de disponer de los medios necesarios para atender al alumnado con necesidades específicas de apoyo educativo (dotando de profesionales especializados, medios y materiales, flexibilidad organizacional en los centros o elaboración de adaptaciones y diversificaciones precisas, entre otros).

Si nos adentramos en la legislación educativa española, se define al alumnado con necesidades educativas como aquél que en función de sus características personales, familiares, ambientales y sociales, necesita de una atención educativa diferente a la ordinaria, y categoriza a este alumnado en diferentes categorías, en función de las características y necesidades del alumnado.

Vivat Academia. Revista de Comunicación. 2021, nº 154, 1-19 


\section{LOMCE}

Se expone (en el artículo 71 de la LOMCE) la necesidad de potenciar el máximo desarrollo personal, intelectual, social y emocional del alumnado a través de la dotación de recursos para la atención educativa de esta tipología de alumnado. Por ello, las medidas de atención a la diversidad han de estar incluidas dentro de la organización escolar, realizándose las adaptaciones y diversificaciones curriculares precisas que faciliten la adquisición y consolidación de los aprendizajes correspondientes.

Dentro de las medidas contempladas en la LOMCE, encontramos dos niveles de concreción en la atención educativa del alumnado: medidas generales para todo el alumnado y medidas específicas según las características del alumnado y de su apoyo educativo.

A nivel general del alumnado, la LOMCE establece principios, como los expuestos anteriormente, que ejerzan como elemento compensador de las desigualdades, así como valores de solidaridad, tolerancia, igualdad y respeto para la superación de la discriminación. Se expone la necesidad de adecuar la educación a la diversidad de aptitudes, intereses, expectativas y necesidades del alumnado.

Para ello, a lo largo del articulado de las diferentes etapas, se enfatiza en la necesidad de adaptar los procesos de enseñanza y aprendizaje a los ritmos de trabajo de los alumnos, así como desarrollar sus capacidades afectivas en todos los ámbitos, sus relaciones con los demás y educar en la no violencia. Este planteamiento, parte de la necesidad de establecer el aprendizaje como un elemento común para los alumnos, sin que las características personales de los mismos supongan un elemento discriminatorio para su acceso en igualdad de oportunidades. El desarrollo legislativo pone especial atención al Diseño Universal para el Aprendizaje (DUA) como elemento que permite la adecuación de la enseñanza a todo el alumnado, a través de una mayor flexibilización del currículo y de los medios y materiales educativos (Alba, 2016).

Como medidas para la atención a la diversidad del alumnado en general, se establecen estrategias de flexibilización y adecuación de la enseñanza como la adaptación curricular, la integración de materias en ámbitos, los agrupamientos flexibles, el desdoblamiento en grupos, la creación de programas de refuerzo o los programas individualizados para el alumnado con necesidades específicas de apoyo educativo (ACNEAE). Estas medidas, enfocadas principalmente para el alumnado perteneciente a la etapa de Educación Secundaria Obligatoria, se organizan alrededor de la autonomía pedagógica de los centros en cuanto a su organización y adopción de medidas de atención a la diversidad.

El planteamiento educativo se centra en la adquisición de las competencias clave como condición indispensable para lograr el pleno desarrollo personal, social y profesional del alumnado, a través del enfoque competencial. Se expone la posibilidad de que los centros educativos puedan elaborar la propuesta curricular

Vivat Academia. Revista de Comunicación. 2021, nº 154, 1-19 


\section{LOMCE}

adaptando el currículo oficial a las necesidades educativas diferenciales del alumnado y garantizando la cobertura de sus necesidades y la promoción de su autonomía.

De manera complementaria, la LOMCE establece una categorización del alumnado con necesidades educativas especiales (que pasaremos a especificar en las medidas de atención específicas del alumnado). Si tomamos en cuenta la importancia de los principios de inclusión educativa, accesibilidad universal y calidad de la educación para todo el alumnado (establecido en el artículo 1 de la LOMCE), podemos vislumbrar un amplio abanico de respuestas educativas para la diversidad del alumnado que, bajo la normativa nacional, supone el planteamiento general de principios metodológicos y pedagógicos que han de ser concretados bajo el marco autonómico y que siguen los planteamientos de las directrices internacionales respecto a la atención educativa del alumnado, como la Declaración de Incheon (2015) o los Derechos de la Infancia (1989).

Respecto a las medidas de atención específicas del alumnado con necesidades educativas especiales, el artículo 71 de la LOMCE categoriza al alumnado en principales grupos de atención educativa en función de sus necesidades personales (alumnado con necesidades educativas especiales derivadas de discapacidad o trastornos graves de conducta, alumnado con trastorno por déficit de atención con/sin hiperactividad -TDAH-, altas capacidades, incorporación tardía y condición personal o historia escolar).

Las principales medidas establecidas pasan por una identificación y valoración temprana de sus necesidades de aprendizaje bajo los principios educativos anteriormente mencionados. A nivel general, para esta tipología de alumnado, se establecen la dotación de recursos de atención educativa y el establecimiento de procedimientos y recursos que identifiquen sus necesidades y garanticen su escolarización y la participación familias, así como la adopción de medidas que conllevan un asesoramiento especializado.

A nivel concreto, podemos encontrar medidas a nivel escolarización (en centros o unidades de educación especial, para el alumnado con necesidades educativas especiales), la flexibilización del currículo escolar mediante adaptaciones curriculares (para el alumnado con necesidades educativas especiales, que presente TDAH y/o el alumnado con dificultades específicas de aprendizaje), planes y programas de enriquecimiento curricular, así como medidas de flexibilización en la duración de las etapas educativas (para el alumnado que presenta altas capacidades), escolarización en función de sus circunstancias, conocimientos, edad e historial académico así como programas específicos a nivel lingüístico y de refuerzo de las competencias o conocimientos básicos (para el alumnado de incorporación tardía en el sistema educativo español), programas específicos de educación compensatoria (para aquellos alumnos que se encuentren en situaciones desfavorables derivadas de factores personales, sociales, económicos, culturales, geográficos, étnicos o de otra índole). 


\section{LOMCE}

Estas medidas concretas de atención a la diversidad (LOMCE, 2013), se concretan a nivel autonómico en virtud de la transferencia de competencias educativas y de atención a la diversidad a las Comunidades Autónomas, aunque todas estas medidas autonómicas han de partir y respetar los principios y fines de atención a la diversidad establecidos en la normativa educativa nacional.

Como hemos indicado anteriormente, la situación en la que se encuentra el sistema educativo actualmente ha necesitado de la concreción de instrucciones específicas para la continuación de la enseñanza en entornos virtuales de aprendizaje. Por ello, el Ministerio de Educación y Formación Profesional ha dictado un marco de actuación y directrices de actuación para el tercer trimestre del curso 2019-2020 y el inicio del curso siguiente, ante la situación de crisis social y educativa provocada por la COVID-19 (BOE, 2020b).

Las directrices generales establecidas por dicha Orden se dividen en siete ejes principales. Respecto al cuidado de las personas, se considera un principio fundamental debido a la situación sobrevenida y se establece el apoyo a los estudiantes y sus familias para hacer posible el desarrollo de sus tareas y funciones, siempre bajo el precepto y cumplimiento de los derechos de la Convención de los Derechos de la Infancia de la ONU.

Respecto al curso escolar, la adaptación de las actividades académicas al alumnado con el objetivo de que se mantengan incorporados al aprendizaje continuo en función de su edad, características y situación excepcional que estén viviendo, suponen una directriz general a seguir. Se complementa la actividad educativa durante el periodo académico a través de la realización de actividades de refuerzo en periodo estival, coordinado mediante entes de la comunidad educativa (entidades locales, asociaciones u organizaciones).

El alumnado que, por su situación personal o sobrevenida, se encuentre desconectado o no localizable, contará con medidas y planes específicos de recuperación del vínculo escolar y de refuerzo, para asegurar su reincorporación a la actividad educativa. Adicionalmente, se pone énfasis en la identificación de las carencias tecnológicas y digitales del alumnado y su estimulación en el uso, a través de la dotación de dichos recursos.

Un aspecto primordial, es la atención al alumnado con necesidad específica de apoyo educativo. Se establece la necesidad de la organización de los recursos de apoyo de los centros educativos para favorecer el acceso al currículo a través de la adaptación de los instrumentos, tiempos y apoyos.

El aprendizaje se centra en los aprendizajes y competencias imprescindibles de la etapa, a través de la flexibilización de los planes de trabajo y desarrollando actividades de recuperación, repaso, refuerzo y ampliación de los contenidos vistos, 


\section{LOMCE}

y bajo un diseño globalizador e interdisciplinar, promoviendo el trabajo cooperativo de los docentes que imparten clase dentro de un mismo grupo-clase de alumnos.

La evaluación se ha de adaptar a la situación del alumnado, no perjudicando al alumnado en su formación debido a las características extraordinarias de la situación, destacando la evaluación diagnóstica y formativa del alumnado (para la elaboración de planes de refuerzo y recuperación necesarios). La promoción del alumnado se considera la norma general, siendo la repetición una medida extraordinaria de aplicación, siempre y cuando el alumno no haya alcanzado los objetivos de etapa ni haya desarrollado convenientemente las competencias establecidas.

Las medidas de atención a la diversidad, individuales o grupales, que den respuesta a las necesidades educativas concretas de los alumnos y a la consecución de los resultados de aprendizaje vinculados a los aprendizajes imprescindibles serán el punto de partida para la configuración del curso educativo 2020-2021.

Estas directrices establecidas por el Ministerio de Educación y Formación Profesional suponen el punto de partida para las Comunidades Autónomas y los centros educativos, en virtud de sus competencias establecidas en la LOMCE respecto a la planificación y organización educativa. Como hemos citado anteriormente, se alude a la autonomía de los centros para establecer la planificación educativa durante este periodo excepcional educativo y se establecen mecanismos que apalien la situación actual.

Si tomamos en consideración las medidas de actuación generales y específicas respecto al alumnado con necesidades específica de apoyo educativo y las directrices establecidas por la Orden EFP/365/2020, podemos ver que la atención a la diversidad del alumnado en situaciones excepcionales educativas se considera un aspecto ha tener en cuenta, pero son los centros los que han de establecer los mecanismos pertinentes para que el alumnado reciba los apoyos necesarios.

\section{METODOLOGÍA}

\subsection{Objetivos.}

Esta investigación tiene como objetivo principal analizar las medidas de atención a la diversidad expuestas en la legislación educativa nacional actual y su aplicación en la situación especial de aprendizaje que vivimos a partir del cierre de los centros educativos provocado por el COVID-19. Para alcanzar este objetivo, hemos planteado los siguientes objetivos:

1. Poner en claro el concepto profundo de atención a la diversidad según la LOMCE.

2. Profundizar en las medidas de atención educativas de la Orden EFP/365/2020 y su coherencia con la legislación actual.

3. Analizar la aplicación de las medidas de atención a la diversidad, expuestas en la legislación educación vigente, dentro de la configuración actual del

Vivat Academia. Revista de Comunicación. 2021, nº 154, 1-19 


\section{LOMCE}

sistema educativo (digitalización de la enseñanza, virtualización de los espacios educativos, etc.).

\subsection{Contextualización.}

Debido a la naturaleza, el estado del arte y de la literatura científica (Cáceres, 2020; Casanova, 2020; Díez-Gutiérrez \& Gajardo-Espinoza, 2020; Feito, 2020; Moreno, 2020; Sainz \& Sanz, 2020) y objetivos del trabajo, la metodología, de corte cualitativa, se ha organizado a partir de la búsqueda, análisis, selección y referenciación de los documentos y fuentes fundamentales sobre la atención a la diversidad del alumnado y la enseñanza durante la COVID-19. Los documentos y fuentes seleccionados corresponden a tres categorías principales:

Tabla 1. Categorías de los documentos y fuentes seleccionadas.

\begin{tabular}{|l|l|l|}
\hline $\begin{array}{l}\text { Documentos e } \\
\text { información de } \\
\text { instituciones privadas }\end{array}$ & Publicaciones oficiales & Literatura científica \\
\hline $\begin{array}{l}\text { Blog de centros de } \\
\text { investigación privados }\end{array}$ & $\begin{array}{l}\text { Boletín Oficial del } \\
\text { Estado }\end{array}$ & $\begin{array}{l}\text { Véase apartado de } \\
\text { bibliografía }\end{array}$ \\
\hline Fundación COTEC & $\begin{array}{l}\text { Organización para la } \\
\text { Cooperación y el } \\
\text { Desarrollo Económicos }\end{array}$ & \\
\hline UNICEF & $\begin{array}{l}\text { Ministerio de } \\
\text { Educación y } \\
\text { Formación Profesional }\end{array}$ & \\
\hline
\end{tabular}

Fuente: Elaboración propia.

A la hora de la selección de la bibliografía, se han utilizado bases de datos nacionales e internacionales, donde nos hemos encontrado con dificultades para la obtención de resultados relevantes. Se ha utilizado un total de 10 bases de datos para la obtención de resultados bibliográficos, relativos a la temática y a los objetivos del presente trabajo.

Tabla 2. Resultados de búsqueda bibliográfica.

\begin{tabular}{|l|l|l|l|}
\hline Base de datos & $\begin{array}{l}\text { Término } \\
\text { "Educación } \\
\text { COVID" }\end{array}$ & $\begin{array}{l}\text { Término } \\
\text { "Atención } \\
\text { diversidad } \\
\text { COVID" }\end{array}$ & $\begin{array}{l}\text { Término } \\
\text { "Educación } \\
\text { inclusiva } \\
\text { COVID" }\end{array}$ \\
\hline $\begin{array}{l}\text { Metabuscador } \\
\text { de la }\end{array}$ & $\begin{array}{l}7614 \text { resultados } \\
17 \text { relevantes }\end{array}$ & $\begin{array}{l}44 \text { resultados } \\
8 \text { relevantes } \\
1 \text { seleccionados }\end{array}$ & $\begin{array}{l}10 \text { resultados } \\
\text { Universidad de } \\
\text { Castilla-La seleccionados }\end{array}$ \\
$\begin{array}{l}\text { Mancha } \\
\text { (PLINIO) }\end{array}$ & seleccionados & \\
\hline REBIUN & 0 resultados & $\begin{array}{l}0 \text { resultados } \\
0 \text { relevantes }\end{array}$ & $\begin{array}{l}0 \text { resultados } \\
0 \text { relevantes }\end{array}$ \\
\hline
\end{tabular}


Rodríguez Torres, J. y Gómez Jiménez, O.

La atención a la diversidad durante la COVID-19: revisión legislativa de las medidas según LOMCE

\begin{tabular}{|l|l|l|l|}
\hline & 0 seleccionados & 0 seleccionados & 0 seleccionados \\
\hline DIALNET & 322 resultados & 54 resultados & 19 resultados \\
& 12 relevantes & 10 relevantes & 5 relevantes \\
& 0 seleccionados & 0 seleccionados & 0 seleccionados \\
\hline EBSCHOHOST & 57 resultados & 0 resultados & 365 resultados \\
& 1 relevantes & 0 relevantes & 28 relevantes \\
& 1 seleccionados & 0 seleccionados & 1 seleccionados \\
\hline WORLDCAT & 18 resultados & 0 resultados & 0 resultados \\
& 5 relevantes & 0 relevantes & 0 relevantes \\
& 3 seleccionados & 0 seleccionados & 0 seleccionados \\
\hline SCHOOLAR & 1440 resultados & 441 resultados & 177 resultados \\
GOOGLE & 75 relevantes & 35 relevantes & 24 relevantes \\
& 9 seleccionados & 2 seleccionados & 3 seleccionados \\
\hline PROQUEST & 85 resultados & 0 resultados & 35 resultados \\
& 3 relevantes & 0 relevantes & 8 relevantes \\
& 1 seleccionados & 0 seleccionados & 1 seleccionados \\
\hline PUBPSYCH & 0 resultados & 0 resultados & 0 resultados \\
& 0 relevantes & 0 relevantes & 0 relevantes \\
& 0 seleccionados & 0 seleccionados & 0 seleccionados \\
\hline WEB & 56 resultados & 0 resultados & 0 resultados \\
SCIENCE & 3 relevantes & 0 relevantes & 0 relevantes \\
& 0 seleccionados & 0 seleccionados & 0 seleccionados \\
\hline SCOPUS & 208 resultados & 0 resultados & 0 resultados \\
& 9 relevantes & 0 relevantes & 0 relevantes \\
& O seleccionados & seleccionados \\
\hline
\end{tabular}

Fuente: Elaboración propia.

\subsection{Instrumento.}

Del total de 30 obras seleccionadas para la realización de este trabajo, solamente 10 han sido utilizados, dado que se ha encontrado que no existe documentación previa asociada a la línea temática que se presenta. Las conclusiones obtenidas se han llevado a cabo a través del análisis de la legislación educativa vigente, la revisión de la conceptualización de la atención a la diversidad y de sus medidas de atención educativa, y los resultados obtenidos del proceso de revisión bibliográfica.

El método de trabajo utilizado se fundamenta en el análisis de los contenidos, es decir, la forma de investigar consiste en captar, evaluar, seleccionar y sintetizar los mensajes implícitos y explícitos en una serie de documentos (Dulzaides y Molina, 2004). De esta forma la descripción y la interpretación de los textos a estudio se ha convertido en el eje principal de la investigación (Bardin, 2002), en el caso que nos ocupa y siguiendo el objetivo del trabajo, se han tenido en cuenta como unidad de análisis:

1. La atención a la diversidad:
a. Conceptualización
b. Medidas legislativas
c. Implicaciones educativas

2. Modelo educativo

a. Recursos y estrategias 


\section{LOMCE}

b. Tipología de atención educativa

c. Modelo de educación actual

d. Características del alumnado

\subsection{Procedimiento.}

El método de trabajo utilizado se fundamenta en el análisis de los contenidos. Partiendo de nuestros intereses, naturaleza del tema y emergencia de algunas evidencias nos lleva a considerar que trabajar con fuentes documentales es la mejor manera de conseguir nuevos conocimientos sobre el tema en cuestión. El procedimiento de trabajo ha consistido en interrogar a los distintos documentos con interpelaciones implícitas, es decir, siguiendo a Ruiz e Ispizua, (1989) utilizar la lectura como una mezcla de entrevista/observación pudiéndose desarrollar como cualquiera de ellas. Las fases de la metodología seguida se resumen en:

1. Definir el universo del material documental. Para la definición del universo material documental, se ha realizado una selección de las categorías de los documentos y de las fuentes seleccionadas durante quince días.

2. Seleccionar una muestra. Debido a la temática concreta de este trabajo, se ha establecido una búsqueda bibliográfica extensa, a través de diferentes fuentes, para la concreción de la temática del trabajo, durante un tiempo total de veinte días.

3. Definir las unidades de análisis básicas que se van a recoger (palabras, frases, párrafos, textos completos, imágenes). La selección bibliográfica responde a la temática concreta del trabajo que se presenta, estableciendo una selección de las obras por fecha de publicación y por la relación directa con la temática de estudio. Dicho proceso tuvo una duración de diez días.

4. Codificar de las categorías conceptuales. Para la codificación de las categorías conceptuales, se comenzó por el análisis de las fuentes legislativas, las cuales establecieron el patrón de codificación de las fuentes bibliográficas. Este proceso tuvo una duración de veinte días.

5. Hacer un pretest de la codificación hecha. Esta fase, cuya duración fue de diez días, permitió seleccionar correctamente la bibliografía utilizada y establecer los elementos del análisis.

6. Realizar el análisis. El análisis de la documentación bibliográfica se realizó de manera exhaustiva, dando respuesta a hipótesis planteadas en los objetivos del trabajo, con una duración del análisis de veinte días.

\section{DISCUSIÓN}

La construcción del nuevo escenario educativo en el que la comunidad escolar se encuentra inmersa depende de dos factores principales: la autonomía de los centros para establecer las medidas educativas necesarias y la alfabetización mediáticoinformacional de las familias (Trujillo, 2020).

A la hora de establecer las medidas, es necesario tener en cuenta diversos factores como: el acceso a Internet en el hogar familiar, la competencia de las familias (tanto 


\section{LOMCE}

digital como cultural) y la situación personal y familiar del alumnado. Las familias con más necesidades carecen de condiciones materiales, culturales, pedagógicas, formativas e, incluso, emocionales para poder enfrentar la situación personal y familiar derivada de una paralización social y económica.

Diez y Gajardo (2020) indican que la brecha social y socioeconómica que sufre gran parte de la población se ve potenciada ante una situación de crisis y de confinamiento. A esta brecha social se suma la brecha digital y tecnológica, aumentando más aún la desigualdad. A pesar de los esfuerzos en la dotación de recursos tecnológicos (por parte de las Administraciones Educativas y los centros escolares), la analfabetización digital hace necesario replantear los procesos de enseñanza y aprendizaje actuales, en pro de la búsqueda de metodologías que favorezcan el aprendizaje y tengan en cuenta el grado de autonomía y desarrollo del alumnado (Moreno, 2020).

El impacto de la situación educativa actual va a tener un efecto desigual entre al alumnado, ya que los apoyos académicos, los recursos tecnológicos, las habilidades no cognitivas y el nivel cultural familiar supone un hándicap para los alumnos más desfavorecidos que resulta difícil de suplir a través de la formación virtual.

A pesar de establecer adaptaciones curriculares o medidas compensatorias para aquel alumnado que pueda encontrarse en situación de desconexión tecnológica o que, por sus características personales, necesite de una individualización de la enseñanza, la enseñanza virtual requiere que el alumnado tenga fuerza de voluntad y cuente con apoyo familiar cualitativo para su correcta implementación (Feito, 2020).

Como hemos visto, la legislación educativa actual, no establece en su articulado medidas de atención al alumnado en situaciones de emergencia sociales o educativas $y$, el articulado de sus medidas tan solo expone situaciones o escenarios genéricos para la atención del alumnado, que han de ser concretados en función de los contextos sociales y educativos en los que se plantee su aplicación.

El actual modelo educativo se centra en la adquisición de las competencias clave (como hemos indicado anteriormente) a través de un enfoque competencial que conlleve cambios escolares para asegurar que el alumnado alcance su pleno desarrollo (BOE, 2015). La integración de las competencias en el sistema educativo conlleva el cambio del foco de la educación hacia la atención del alumnado y de sus posibilidades de aprendizaje, intentando maximizar el desarrollo de las capacidades y de los aprendizajes de estos (Rodríguez, 2010).

Los planteamientos educativos durante el confinamiento social se pueden dividir en dos vertientes principales (Díez y Gajardo, 2020): un planteamiento orientado hacia el trabajo de las competencias básicas basado en el acompañamiento tutorial y un segundo planteamiento centrado en la continuación del proceso de enseñanza y aprendizaje según lo establecido en las programaciones didácticas adecuado al

Vivat Academia. Revista de Comunicación. 2021, nº 154, 1-19 


\section{LOMCE}

contexto virtual actual del aprendizaje. Ambos planteamientos han de ser analizados a partir del contexto socioeconómico y cultural de los centros, analizando las posibilidades tecnológicas y personales del alumnado de los centros.

El desarrollo normativo establece la priorización del trabajo competencial del alumnado, estableciendo una categorización, por parte de los docentes, de las competencias a trabajar. Complementariamente, el trabajo cooperativo del tándem familia-escuela es clave para alcanzar los objetivos de aprendizaje (Cáceres, 2020). Esta situación excepcional pone a prueba la fortaleza de nuestro sistema educativo, y sobre todo de la respuesta que damos a la atención de las necesidades de aprendizaje de nuestro alumnado.

Las actividades de enseñanza a través de medios tecnológicos deben de respetar el desarrollo competencial del alumnado y promover el aprendizaje de valores intrapersonales y sociales necesarios para afrontar las situaciones sociales extraordinarias. Deben de partir de un diseño accesible para todo el alumnado (independientemente de sus posibilidades de acceso al entorno virtual) y deben de dar respuesta a las carencias competenciales que puede llegar a presentar el alumnado (como aquellas con las que nos encontramos a la vuelta de los periodos estivales de larga duración, como las vacaciones de verano), ya que un retroceso en el aprendizaje del alumnado supondría un incremento en la tasa de repetición y no promoción (Sainz y Sanz, 2020).

Haciendo alusión a la necesaria coordinación y cooperación entre la comunidad educativa y los centros escolares, Saldaña, Álvarez, Aguilera, Moreno, Moreno y de los Reyes (2008) exponen que es necesaria la inclusión, dentro de la organización educativa, de entidades externas de apoyo (como entidades de apoyo a personas de discapacidad), ya que permiten dar cumplimiento a las necesidades de individualización de la enseñanza y revierte positiva en el sistema educativo a través de la suplencia de las carencias profesionales del sistema educativo, ofrece formación especializada e individualizada sobre las necesidades educativas del alumnado al que se presta apoyo y dota de recursos personales y materiales a los centros educativos.

Adicionalmente, el principio de colaboración familiar requiere de un trabajo coordinado entre docentes y núcleos educativos de los alumnos y alumnas. Situaciones excepcionales, como la actual, suponen un aumento del tiempo de convivencia familiar (tanto a nivel cuantitativo como cualitativo) y revierten en una mejora de la atención individual de las familias hacia sus hijos e hijas. Para ello, y con el objetivo de dar continuidad al proceso de aprendizaje, se hace imprescindible la creación de canales eficaces de comunicación entre docentes y familias, donde se prime la salud emocional, las habilidades intra e interpersonales y la convivencia pacífica (estableciendo en un segundo nivel de importancia el seguimiento de los contenidos académicos)

\section{CONCLUSIÓN}




\section{LOMCE}

Una situación tan excepcional como la actual, supone una inigualable ocasión para mejorar transformando la escuela (Feito, 2020). Contamos con planteamientos teóricos, investigaciones marco sobre como ha de ser la aplicación didáctica, multitud de recursos y estrategias con sobrenombres varios... Un sinfín de materiales que son aplicados en el día a día de los centros, pero que no están preparados para la excepcionalidad. Así como tampoco los docentes están preparados para un cambio del modelo educativo.

Llegados a este punto, conviene destacar que nuestro sistema educativo (siguiendo la regla de otros muchos a nivel mundial), no encuentra escenarios planificados para situaciones excepcionales sociales y que, sus planteamientos sobre la atención a la diversidad se centran en propuestas teóricas difícilmente de ser llevadas a cabo durante la situación social provocada por la COVID-19. Nos encontramos en un momento educativo donde es necesario centrar los esfuerzos en acompañar y tutorizar al alumnado por encima de la continuación de las planificaciones docentes, donde la evaluación corresponde al seguimiento emocional de nuestro alumnado y la herramienta educativa más eficaz es la empatía y la cercanía personal.

Meirieu (2020) expone que lo importante que es "hacer el aula" para "hacer la escuela", donde los alumnos se sientan parte del todo y las desigualdades no existan en beneficio de su desarrollo personal. La digitalización forzada que ha derivado de la situación social lleva a replantear la necesidad de reorganizar la enseñanza virtual en pro de su uso en el conjunto del alumnado, ya que los principios básicos del sistema educativo no son respetados por las desigualdades provocadas por la brecha digital.

Utilizar experiencias cotidianas del entorno familiar para el desarrollo de actividades de aprendizaje basadas en el desarrollo de las competencias, la reorganización del currículo educativo o el asesoramiento familiar individualizado, son medidas ha tener en cuenta a la hora de establecer las planificaciones docentes en situaciones excepciones de aprendizaje.

La educación y los centros escolares son un lugar de protección y reducción de las desigualdades sociales, donde los alumnos encuentran normalidad, estabilidad y esperanza (UNICEF, 2020). El sistema educativo, como hemos visto, ofrece un sistema de protección presencial para el conjunto del alumnado, pero los principios inclusivos y las medidas de atención a la diversidad quedan relegados a un segundo plano en situaciones excepcionales de emergencia social (como la provocada por el COVID-19).

Apelar a la autonomía de los centros y a la función tutorial del profesorado para crear herramientas y planes específicos de protección educativa, sumado a una brusca adaptación hacia un sistema educativo nuevo y no estructurado, supone la omisión de la atención educativa individualizada del alumnado, ya que la premura 


\section{LOMCE}

situacional y la accesibilidad general del conjunto educativo suponen el conjunto de los esfuerzos actuales.

Nuestro sistema educativo se encuentra en un momento de encrucijada social, donde todo lo establecido no es llevado a cabo, aumentando la brecha social y estableciendo una nueva categorización del alumnado en función de sus recursos y competencias digitales. Las medidas de atención a la diversidad dictadas hasta el momento no permiten solventar las carencias de aprendizaje con las que se encuentran aquellos alumnos descolgados del sistema educativo, ya que el énfasis se encuentra en la continuación o no de las programaciones docentes y la promoción del alumnado, obviando los impactos negativos que supone la situación a nivel social y escolar.

Como expone Casanova (2020), se pueden llevar a cabo los planteamientos inclusivos que estructuran nuestra normativa educativa base, ya que tan solo requiere de tomar las mejores decisiones con los recursos existentes para ajustar el modelo educativo a las prioridades del alumnado.

\section{REFERENCIAS}

Alba, C. (coord.) (2016). Diseño universal para el aprendizaje. Madrid: Morata.

Bardin, L. (2002). Análisis de contenido. Madrid: Akal.

Barton, L. (2009). Estudios sobre discapacidad y la búsqueda de la inclusividad. Observaciones. Revista de Educación, 349, 137-152. https://cutt.ly/FyR66TA

BOE. (2006, 4 de mayo). Ley Orgánica 2/2006, de 3 de mayo, de Educación. Boletín Oficial del Estado. https://cutt.ly/fyR6MV7

BOE (2010, 6 de abril). Orden EDU/849/2010, de 18 de marzo, por la que se regula la ordenación de la educación del alumnado con necesidad de apoyo educativo y se regulan los servicios de orientación educativa en el ámbito de la gestión del Ministerio de Educación, en las ciudades de Ceuta y Melilla. Boletín Oficial del Estado. https://cutt.ly/nyR6NXQ

BOE. (2013, 10 de diciembre). Ley Orgánica 8/2013, de 9 de diciembre, para la mejora de la calidad educativa. Boletín Oficial del Estado. https://cutt.ly/VyR6NwP

BOE. (2015, 29 de enero). Orden ECD/65/2015, de 21 de enero, por la que se describen las relaciones entre las competencias, los contenidos y los criterios de evaluación de la educación primaria, la educación secundaria obligatoria y el bachillerato. Boletín Oficial del Estado. https://cutt.ly/fyTqyvG 


\section{LOMCE}

BOE. (2020a, 14 de marzo). Real Decreto 463/2020, de 14 de marzo, por el que se declara el estado de alarma para la gestión de la situación de crisis sanitaria ocasionada por el COVID-19. Boletín Oficial del Estado. https://cutt.ly/JyphL2L

BOE. (2020b, 22 de abril). Orden EFP/365/2020, de 22 de abril, por la que se establecen el marco y las directrices de actuación para el tercer trimestre del curso 2019-2020 y el inicio del curso 2020-2021, ante la situación de crisis ocasionada por el COVID-19. Boletín Oficial del Estado. https://cutt.ly/cyTquJf

Cáceres, K. F. (2020). Educación virtual: creando espacios afectivos, de convivencia y aprendizaje en tiempos de COVID-19. CienciAmericana, Número Especial Desafíos Humanos ante el COVID-19, abril-octubre, 9(2). http://dx.doi.org/10.33210/ca.v9i2.284

Casanova, M. A. (2020, 28 de marzo). La educación inclusiva (más allá de la discapacidad) se complica en tiempos de virus. theconversation.com. https://cutt.ly/OyYgpj0

Cueto García, C. (2020). El COVID19 acelera la digitalización de los profesores. Computerworld. https://cutt.ly/IyTqeI3

Diez-Gutierrez, E. \& Gajardo-Espinoza, K. (2020). Educar y Evaluar en Tiempos de Coronavirus: la Situación en España. Multidisciplinary Journal of Educational Research, 10(2), 102-134. http:/ / dx.doi.org/10.4471/remie.2020.5604

Dulzaides, M. E. y Molina, A.M. (2004). Análisis documental y de información: dos componentes de un mismo proceso. ACIMED: Revista Cubana de Información en Ciencias de la Salud, 12(2), 1. https:/ / cutt.ly/0yTqh9W

EFE (2020). El Gobierno recomienda el cierre de los colegios en todas las comunidades. Agencia EFE. https://cutt.ly/IyTqiEh

Espinosa, D., Reyes, M., \& Rodríguez, L. (2015). Inclusión y Educación. https://cutt.ly/5yTqqA3

Feito, R. (2020). Este es el fin de la escuela tal y como la conocemos. Unas reflexiones en tiempo de confinamiento. Revista de Sociología de la Educación-RASE, 13(2) Especial, COVID-19, 156-163. http://dx.doi.org/10.7203/RASE.13.2.17130.

Juste, M. (2020). Coronavirus: Grandes y pequeñas empresas ofrecen gratis sus plataformas de educación online. Expansión. https://cutt.ly/jyTqr8b

Martín, J. M., \& Rogero, J. (2020). El coronavirus y la asfixia educativa: el confinamiento deja sin protección a la infancia más vulnerable. eldiario.es. https://cutt.ly/GyTqwR4 


\section{LOMCE}

Moreno, S. M. (2020). La innovación educativa en los tiempos del coronavirus. Salutem Scientia Spiritus, 6(1), 14-26. https://cutt.ly/kyR6LUK

Rodríguez, J. (2010). De las programaciones didácticas a la unidad didáctica: incorporación de las competencias básicas y la concreción de tareas. Revista Docencia e Investigación, 20, 254-270. https://cutt.ly/kyR6KXB

Rodríguez, J., \& Gómez, O. (2018). Cambio de paradigma, la inclusión educativa. Análisis legislativo y comparativo. En Contenidos universitarios innovadores (1. a ed.). Editorial Gedisa.

Ruiz, J. I. e Ispizua, M. A. (1989). La decodificación de la vida cotidiana: métodos de la investigación cualitativa. Universidad de Deusto.

Sainz, J. y Sanz, I. (2020, 3 de abril). Los efectos del Coronavirus en la educación (I): Las pérdidas de clases y rendimientos educativos desiguales. noticias.minuto5.com. https://cutt.ly/lyTwPLt

Saldaña, D., Álvarez, R. M., Aguilera, A., Moreno, F. J., Moreno, M. y de los Reyes, I. (2008). ¿Cómo contribuyen las asociaciones de familiares a la educación del alumnado con autismo? Infancia y Aprendizaje, 31(1), 67-78. https://doi.org/10.1174/021037008783487110

Trujillo, F. (2020). ¿Evaluación del curso? Escenarios posibles para el futuro inminente de la educación. Otrasvoceseneducacion.org. https://cutt.ly/3yR6ZFJ

UNICEF (2020). La educación frente al COVID-19. Propuestas para impulsar el derecho a la educación durante la emergencia. https:/ / cutt.ly/XyYgchW

\section{AUTORES}

\section{Javier Rodríguez Torres}

Doctor en Pedagogía por la Universidad de Alcalá (España) en la línea de formación del profesorado y TIC. Además de maestro de Educación Infantil y Primaria con varias especialidades, realizó Máster de Psicología Escolar en la Universidad Complutense de Madrid. Miembro del Grupo de Investigación CIBERIMAGINARIOUCLM. (https://ciberimaginario.es). En la actualidad es Director Académico de Economía y Planificación de la Universidad de Castilla - La Mancha, también es coordinador del Máster de Secundaria en el Campus de Toledo y director del postgrado de Discapacidad, Inclusión y Ciudadanía. Nuevos Enfoque (ya en la $5^{\text {a }}$ Ed.). Las líneas de investigación, con respecto a las TIC, son su integración curricular, las diferencias de género en su inclusión y currículo, los discursos de poder -saber que se generan en el uso y aplicación de las tecnologías.

javier.rtorres@uclm.es

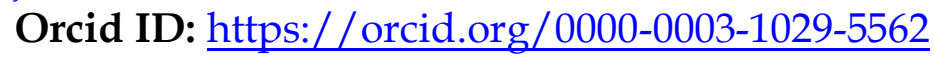




\section{LOMCE}

Google Scholar: https://scholar.google.es/citations?user=CM63_cAAAAAI

ResearchGate: https://www.researchgate.net/profile/Javier_Rodriguez_Torres

Scopus: https://www.scopus.com/authid/detail.uri?authorId=55579914400

Academia.edu: https://uclm.academia.edu/JavierRodriguezTorres

\section{Óscar Gómez Jiménez}

Profesor de Educación Primaria en la especialidad de Inglés y Pedagogía Terapéutica en el Colegio Sagrado Corazón de Jesús y María Inmaculada (Miajadas, Cáceres), Master en Psicopedagogía por la Universidad de Barcelona y doctorando en Investigación en Humanidades, Artes y Educación por la Universidad de Castilla-La Mancha. Especialista en atención a la diversidad y sus líneas de investigación son la legislación educativa, la atención a la diversidad y la educación inclusiva.

osquitagomez@gmail.com

Orcid ID: https://orcid.org/0000-0002-6581-4481

Google Scholar: https://scholar.google.com/citations?hl=es\&user=BtVNhnAAAAAI

ResearchGate: https://www.researchgate.net/profile/Oscar_Gomez20 